\title{
A Two-Ball Mouse Affords Three Degrees of Freedom
}

\author{
I. Scott MacKenzie \\ Computing \& Information Science \\ University of Guelph \\ Guelph, Ontario, Canada \\ $1-519-824-4120$ \\ mac@snowhite.cis.uoguelph.ca
}

\author{
R. William Soukoreff \\ Computing \& Information Science \\ University of Guelph \\ Guelph, Ontario, Canada \\ 1-519-824-4120 \\ will@snowhite.cis.uoguelph.ca
}

\author{
Chris Pal \\ Dept. of Physics \\ University of Guelph \\ Guelph, Ontario, Canada \\ 1-519-824-4120 \\ cpal@uoguelph.ca
}

\begin{abstract}
We describe a prototype two-ball mouse containing the electronics and mechanics of two mice in a single chassis. Unlike a conventional mouse, which senses $x$-axis and $y$ axis displacement only, our mouse also senses $z$-axis angular motion. This is accomplished through simple calculations on the two sets of $x-y$ displacement data. Our mouse looks and feels like a standard mouse, however certain primitive operations are performed with much greater ease. The rotate tool - common in most drawing programs - becomes redundant as objects are easily moved with three degrees of freedom. Mechanisms to engage the added degree of freedom and different interaction techniques are discussed.
\end{abstract}

\section{Keywords}

pointing devices, multi-degree-of-freedom input, rotation

\section{INTRODUCTION}

Since the introduction of the Apple Macintosh in 1984, the form of desktop systems has not changed substantially. Manipulating complex graphic objects usually combines mouse movement with special tools or modes. These permit simple displacement data from the mouse to map to and control the displacement and/or angular location of objects or scenes. These mappings are often unnatural.

\section{DIMENSIONS AND DEGREES OF FREEDOM}

Before we describe our mouse, it is important to distinguish between dimensions (D) and degrees of freedom (df). In three dimensions, there are six degrees of freedom: three for position along the $x, y$, and $z$ axes, and three for angular orientation $(\theta x, \theta y$, and $\theta z)$. In 2D, there are three degrees of freedom. If we consider a $2 \mathrm{D}$ surface such as a mouse pad (see Figure 1), then there is an $x$ and $y$ positional degree of freedom and a $z$-axis rotational degree of freedom.

Figure 2 illustrates the dimensions and degrees of freedom for several "mouse-type" devices. As a standard mouse is manoeuvred on a mouse pad, only its $x$ and $y$ displacement are sensed, as indicated in the "Mouse" column. This is sufficient for most tasks using, for example, word processors or spreadsheets. However, within drawing packages and other graphics programs, a common task is to move an object to a new location and with a new orientation. This is a full two dimensional task and it requires three degrees of freedom. Since mouse angular motion is not sensed, a rotate tool or a manipulator handle is usually required. Although other schemes can be devised to control the orientation of objects [3], they are unnatural and violate the basic perceptual structure of interaction [2].

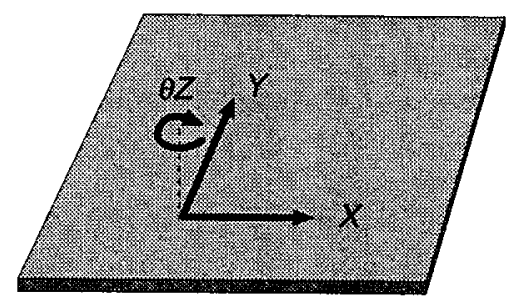

Figure 1. A mouse pad is a two-dimensional surface with three degrees of freedom: $x, y$, and $\theta z$.

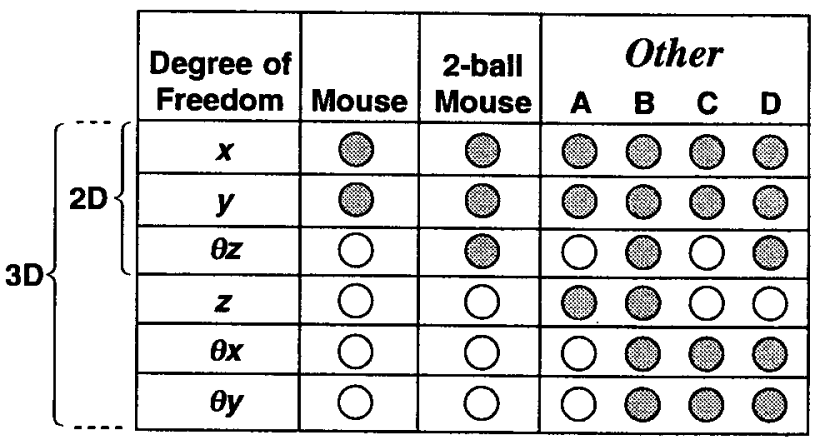

Figure 2. Dimensions vs. degrees of freedom. Grey dots indicate degrees of freedom sensed for several types of input devices. (See text for discussion)

\section{A TWO-BALL MOUSE}

We have built a prototype of a 2D/3df mouse (see Figure 3) that makes a rotate tool redundant. The mouse was built using the mechanical parts from two Microsoft 2.0 mice and the electronics from two Fellowes MousePens. The prototype weighs $156 \mathrm{~g}$, compared to $104 \mathrm{~g}$ for the standard Microsoft 2.0 mouse. It interfaces to a host computer through two serial ports. This is sufficient for a prototype and it allowed a variety of simple demonstrations to be implemented. As a product, a complete bottom-up redesign would be required.

With some simple arithmetic in the interface software, the $z$-axis or rotational component of the mouse's motion is easily computed from the two streams of $x-y$ positional 
data. The result is a mouse that senses all three degrees of freedom in a two-dimensional surface. ("2-ball Mouse" column in Figure 2).

(a)

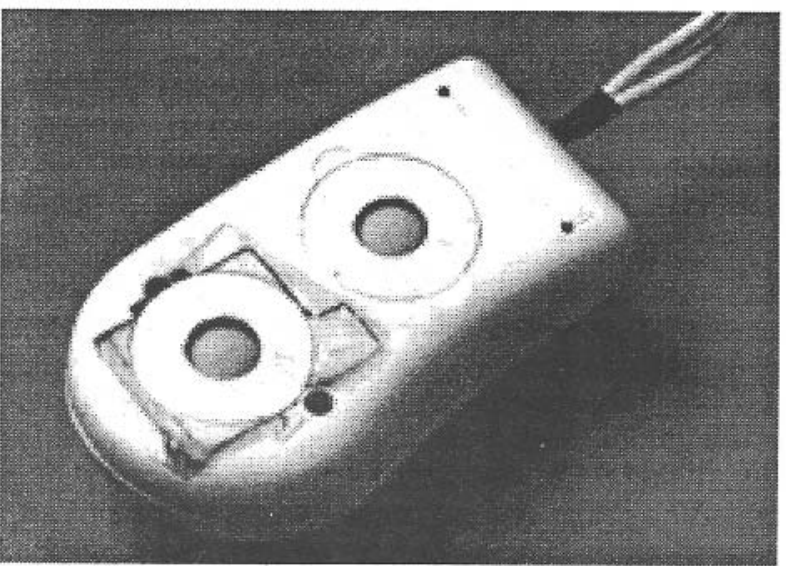

(b)

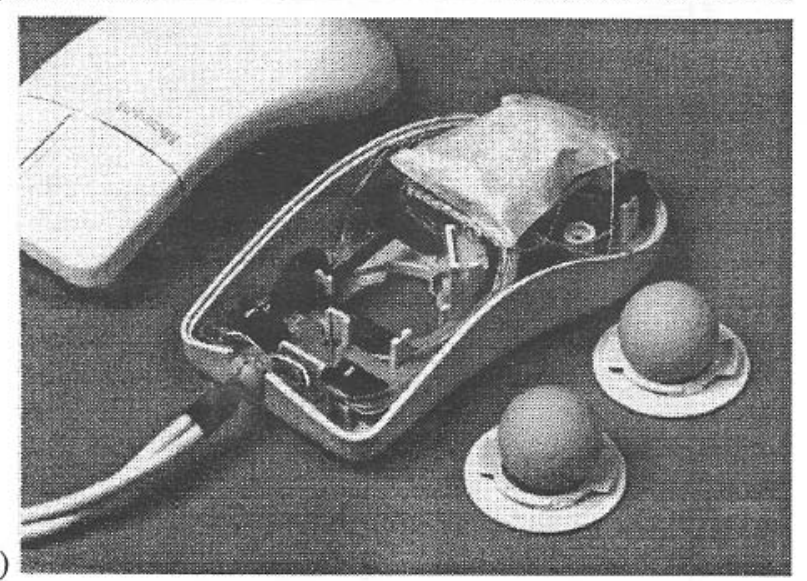

Figure 3. Two-ball mouse (a) bottom view. (b)

top view with cover and balls removed.

MULTI-DEGREE-OF-FREEDOM INPUT DEVICES

There are numerous input devices supporting more than two degrees of freedom. The ProAgio by Mouse Systems and the Intellimouse by Microsoft are 3df mice however they are fundamentally different from our device because rotation is sensed through a roller manipulated by a finger. We characterise these as having $2+1$ degrees of freedom - two positional degrees of freedom, controlled as usual, and a third, but separate, rotational degree of freedom controlled by a finger. Venolia describes a similar device with two rollers [3]. With the frequent occurrence of carpal tunnel syndrome in today's workplace, it is debatable whether increasing the load on the mouse-controlling hand is wise. Our mouse has three integral degrees of freedom making it a natural choice for $2 \mathrm{D} / 3 \mathrm{df}$ interaction.

The $O w l$ by Pegasus Technologies and the AeroDuet by Creative Labs are $3 \mathrm{df}$ pointing devices that are held in the air. They sense $x, y$, and $z$ position so, again, they are distinctly different from our device (" $\mathrm{A}$ " column in Figure 2). There are also a variety of 6df devices, such as the InsideTrak by Polhemus ("B" column in Figure 2). Devices that sense $z$-axis position are operated in the air and are suited to games, virtual reality and other nonmainstream applications. In our view, such devices are not likely to garner the support of users of desktop systems, even if the application leans toward 3D interactive graphics. Current systems that combine a mouse with rotate tools or manipulation handles are familiar and there will be resistance to change unless the benefits are substantial and immediate. Since our device is a mouse, it does not impose a major new technique on the user. And it will work with existing tools as new techniques gain acceptance.

An interesting new device is the Rockin'Mouse [1]. It looks like a mouse except it has a curved bottom allowing it to "rock" on the desktop. The rocking motion yields $\theta x$ an $\theta y$ data ("C" column in Figure 2). It is operated without lifting from the desktop, so it also has that special mouselike appeal. Finally, column "D" in Figure 2 identifies a hypothetical $5 \mathrm{df}$ device. It would sense rocking motion about the $x$ and $y$ axes (like the Rockin'Mouse) as well as $z$-axis angular motion (like our mouse). And it would not require lifting from the desktop. Such a device could be very appealing to users who are reluctant to change their ways.

\section{OPERATION}

That our mouse looks and acts like a regular mouse "most of the time" is important for user acceptance. A problem we faced early on was how to engage the third degree of freedom. In an earlier wooden prototype, this was accomplished using a thumb-operated momentary switch on the side of the chassis. Although this worked, it was awkward and error prone. We finally settled on the following two-handed approach: An object is acquired in the usual way, by positioning the cursor over it and pressing the primary mouse button. If the CTRL or ALT key is also depressed (presumably using the other hand), movement is in $3 \mathrm{df}$ mode; otherwise it is in $2 \mathrm{df}$ mode. This is a simple and natural interaction technique. Using the CTRL key, the hand-to-object angular mapping is 1-to1. Using the ALT key, the mapping is 5-to-1. The latter helps extend the range of rotation beyond that afforded by the biomechanics of the lower arm. We are currently implementing a set of representative tasks for empirical testing and comparison with current practice.

\section{ACKNOWLEDGEMENTS}

We thank NSERC of Canada for supporting this research. Thanks as well to the members of the Input Research Group at the University's of Guelph and Toronto for many helpful suggestions during the development of this research.

\section{REFERENCES}

1. Balakrishnan, R., Baudel, T., Kurtenbach, G., and Fitzmaurice, G. The Rockin'Mouse: Integral 3D manipulation on a plane, In Proc. of $\mathrm{CHI}$ '97. New York: ACM, 1997.

2. Jacob, R. J. K., Sibert, L. E., McFarlane, D. C., and Mullen Jr., M. P. Integrality and separability of input devices, ACM Trans. Computer-Human Interaction 1 (1994), 3-26.

3. Venolia, D. Facile $3 D$ manipulation, In Proc. of INTERCHI '93. New York: ACM, 1993, pp. 31-36. 